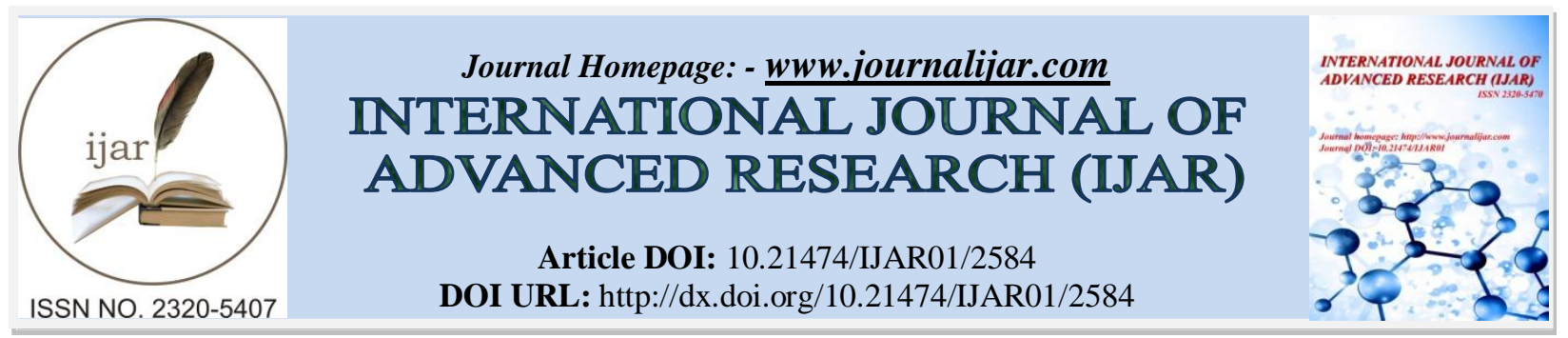

RESEARCH ARTICLE

\title{
ROLE OF KNOWLEDGE, ATTITUDE AND PRACTICE OF LIFESTYLE MODIFICATIONS TOWARDS THE CONTROL OF HBA1C AMONG TYPE 2 DIABETIC PATIENTS IN KING KHALID UNIVERSITY HOSPITAL (KKUH), RIYADH, KINGDOM OF SAUDI ARABIA.
}

\author{
Sulaiman A. Al Shammari ${ }^{1}$, Abdullah K. Alsufyani ${ }^{2}$, Tareq M. Aljurf ${ }^{2}$, Abdullah S. Alkhuraiji ${ }^{2}$, Nasser S. \\ Alsaleh ${ }^{2}$ and Mohammed R. Aldhaheri ${ }^{2}$. \\ 1. Professor, College of Medicine, King Saud University, Riyadh, Saudi Arabia. \\ 2. Medical Student, College of Medicine, King Saud University, Riyadh, Saudi Arabia.
}

\section{Manuscript Info}

Manuscript History

Received: 27 October 2016

Final Accepted: 25 November 2016

Published: December 2016

\section{Abstract}

Introduction: studies have been undertaken in the region concerning diabetes. However, no study was able to provide us with how much info do patients have about the necessity of lifestyle modification in their case and their attitude towards it. We aim in our study to assess knowledge, attitude of patients concerning lifestyle modification necessity their practices and its relation to the progression of their disease.

Methodology: The study was quantitative observational cross sectional study conducted in the Primary Care Clinic, King Khalid University Hospital by the research team using a predesigned questionnaire. The study included all Saudi type - 2 diabetes patients who are over 18 years of age and excluded patients with congestive heart failure, disabilities, mental and communication problems. The required sample size was 104 and patients were selected using Simple Random Sampling technique

Results: Study included 112 respondents, 64 (56.6\%) were males. The mean knowledge score was average (8.7/14) and the mean attitude score was very positive for both females (4.79/5) and males (4.48/5). HbA1c levels were generally poor. One hundred study subjects were overweight, $35 \%$ believed they were not overweight. Only $23(20.4 \%)$ cases of the 112 were following a specific diet. Eighty eight $(77.9 \%)$ cases said they practiced exercise out of which $82(72.6 \%)$ specified walking and $2(1.8 \%)$ swimming.

Conclusion: our study showed that type 2 diabetes mellitus patients in Saudi Arabia have an average amount of knowledge about required lifestyle changes. Attitude to lifestyle changes was extremely good in almost all cases, however exercise among most study individuals turned out to be not so good. This indicates a lack of will-power for the correct implementation of their knowledge and attitude. As for 
HbA1c, levels in the vast majority of cases showed poor control on the disease.

Copy Right, IJAR, 2016,. All rights reserved.

\section{Introduction:-}

Diabetes mellitus is a progressive chronic disease with a rapidly increasing prevalence in both developing and developed countries [1]. It is estimated that there are 415 million diabetes patients globally [2].The Kingdom of Saudi Arabia (KSA) ranked seventh among the top ten countries with high diabetes prevalence [2], and the abnormal glucose metabolism has reached an epidemic level where the prevalence of diabetes was $25.4 \%$ with $40.3 \%$ being unaware of their disease as per what have been reported from the SAUDI-DM study [4]. This might be attributed to the rapid economic development which led to the tremendous changes in lifestyle towards the westernized pattern reflected by changes in nutrition, less physical activity, tendency to increased obesity and more smoking [5, 6]. Diabetes mellitus is well known to be associated with both macrovascular (vasculopathy) and microvascular (retinopathy, nephropathy, and neuropathy) complications [7,8] and is considered to be the eighth leading cause of death worldwide; accounted for 1.5 million (2.7\%) deaths in 2012 [9]. Despite all of these aspects, an apparent gap exists between knowledge and the attitude towards diabetes among diabetic patients [10]. Results of studies from different countries including the GCC ones came out with the conclusion that the diabetes knowledge among diabetic patient is poor and not satisfactory [11- 17].

In regards to what have been previously reported from KSA, one of the earliest studies published in 1999 from Abha and aimed mainly to identify some determinants of compliance with diet, anti-diabetic drugs and the appointment system amongst diabetic patients; came up with the conclusion that only $40 \%$ of patients reported good compliance with their prescribed dietary regimen compared to $47 \%$ who had fair compliance and the compliance was predicted by the degree of care disease duration [12]. Another study undertaken among female diabetic schoolteachers in AlKhobar, Saudi Arabia in 2005 showed that although $87 \%$ of patients considered obesity to be unhealthy, $78 \%$ were actually overweight and obese. They also reported that only $25 \%$ of the patients had compliance with the diet and $5 \%$ did regular exercise [13].

However, due to the fact that the study those previously mentioned studies in KSA were conducted more than 10 years ago, we expect that multiple social and economic factors may have caused significant changes in diabetes patients' attitudes and practices.

Although several studies have been undertaken in the region concerning diabetes, the vast majority of these studies mostly discussed aspects concerning knowledge or attitude of diabetes patient towards diabetes, lifestyle or nutrition in general. Others studied the practice and compliance of the patients. However, no study was able to provide us with how much info do patients have about the necessity of lifestyle modification in their case and their attitude towards it. Taking this into consideration, we aim in our study to assess knowledge, attitude of patients concerning lifestyle modification necessity their practices and its relation to the progression of their disease.

\section{Methodology:-}

\section{Study design and population:-}

This is a quantitative observational cross sectional study that was conducted in the Primary Care Clinic (PCC), King Khalid University Hospital (KKUH), Riyadh, KSA. Data was collected throughout March and April 2014.The study included all Saudi type-2 diabetes patients who were over 18 years of age and excluded patients with congestive heart failure, disabilities, mental and communication problems.

\section{Sample size:-}

The sample size (104 patients) was calculated using the rule of thumb suggested by Green (1991) for regression sample sizes [18]. We suggested a sample size $>104+\mathrm{m}$; for testing individual predictors, where $\mathrm{m}$ is the number of independent variables.

\section{Sampling technique:-}

We used the Simple Random Sampling technique to select from patients visiting the PCC. 


\section{Data collection methods:-}

Data were collected by the research team through interviewing the patients using a predesigned questionnaire (Supplementary 1). The questionnaire included 39 questions, which were categorized into questions for the assessment of knowledge, attitude and practice regarding lifestyle modification. Questionnaire includes knowledge questions taken from the Diabetes Knowledge Test constructed by The Michigan Diabetes Research and Training Center [19], in addition, to other questions assessing attitude and practice based upon the American Diabetes Association recommendations for lifestyle modifications among diabetics.

HbA1c was retrieved from the subject's electronic health record and the Body Mass Index (BMI) was taken from the subject's files. The effect of proposed factors (i.e. Knowledge, attitude, and practice of lifestyle) on HbA1c control among diabetes type-2 patients was analyzed using multinomial logistic regression. Three separate models were created to test the effect of each factor on the HbAlc. The knowledge score was measured by awarding one point for each correct answer and zero for wrong or no response. Then according to the number of points collected, each patient knowledge score was classified into poor (less than 7 points), average (5 to 7 points) and good knowledge (greater than 7 points) [16]. Attitude was constructed using the Likert scale. As in the study from Khan et al. [20], HbAlc results were categorized into good glycemic control ( $\mathrm{HbA} 1 \mathrm{c} \leq 6 \%)$; poor glycemic control $(\mathrm{HbAlc}$ $>6 \%-9 \%)$ and worst glycemic control (HbA1c>9\%).

The study was approved by the Institutional Review Board (IRB) of King Saud University (KSU). An informed consent form was taken from the entire study participant. Permission to access patients' files and lab results was obtained.Confidentiality of the study participants was ensured in addition to the standard of care and fair selection of community and individuals. Identification Numbers (IDs) were assigned to each case to ensure confidentiality. A pilot study was conducted on 20 type 2 diabetes patients to evaluate the validity and visibility of the research questionnaire.

This study is being done for the first time since the introduction of the CMED 305 curriculum in September 2011.

\section{Statistical analysis:-}

Collected data were entered to a computer and were analyzed using SPSS software for statistical analysis. The effect of Patients-related variable on the HbAlc was tested using appropriate statistical tests (Chi-square, t-test, Pearson's correlation).

\section{Results:-}

A total of 190 diabetic patients were asked to participate in this study, of whom 32 patients refused to participate, 46 patients were excluded for matching the exclusion criteria resulting in a final sample size of 112 (Response rate $=$ $59 \%$ ). Males made up 57.14\% (64) of the sample while the remaining $42.86 \%$ (48) were females. The vast majority of cases were: married (91.96\%), living in an urban area (92.86\%), and $62.50 \%$ (70) were in the age group of $40-59$ years old. Of the whole sample, $91.96 \%$ (103)had a BMI $\geq 25$ and most of them (33.93\%) falling into the BMI range of 30-34.9. Majority (67.86\%) of the cases were unemployed and $71.43 \%$ are educated $(24.11 \%$ elementary/secondary, $22.32 \%$ high school/diploma, and $25.00 \%$ university and above) table (1).

Both males and females displayed a similar level of knowledge. However, there was a significant difference among different age groups ( $\mathrm{p}$-value $=0.016$ ). Within the age groups $20-39,40-59$, and 60-78 years old the vast majority of the patients $(62.50 \%, 74.29 \%$, and $81.82 \%$ respectively) had an average level of knowledge, whereas in the age group of 80-99 years old all the patients had poor knowledge. The trend shows an increase in cases with average knowledge compared with a decrease of cases with good knowledge, which is related to the increase in age group. There was no significant relationship between the total knowledge score and HbAlc levels. Educational level and employment status also showed no effect on the knowledge score. (Table 2)

The majority of female cases were unemployed as compared to male, yet isolated unemployed male cases showed no statistical difference in terms of the final knowledge score. The duration of type 2 Diabetes Mellitus did not affect the knowledge score. Educational level also showed no effect on the knowledge score.

Our results also showed an extremely positive attitude towards good diabetes lifestyle practice among Saudi type 2 diabetes mellitus patients. Although both males and females demonstrated positive mean attitude scores for the 
individual questions, females proved to have a significantly more positive attitude than their male counterparts. (Table 3)

Further analysis showed that the duration since diagnosis does not seem to affect the attitude of the cases towards the lifestyle according to our research. Looking at specific questions assessing attitude, it turned out that patients' attitudes towards hydration is not related to their actual water intake. Similar outcomes have been found when comparing attitudes towards exercise and their practice times where there is no relation.

Almost 92\% (103) of the participant had a BMI $\geq 25 \mathrm{~kg} / \mathrm{m} 2$. Amongthem , 35\% believed that they were not overweight. In general, males had significantly higher water intake than females.

There was no difference between the number of cases who follow a specific diet between male and female subjects. Majority of the subjects (79\%) didn't report following a specific diet which was more common in females (85.4\%). Of the study sample, only $23(20.4 \%)$ cases followed a specific diet. Ninety percent of the cases said that they did not calculate their calories and only $4(3.5 \%)$ cases were able to report a specific number for their daily calorie intake.

The majority of our study cases turned out to have poor or at worst HbA1c levels. There was a significant relation between the level of education and related HbA1c levels. Participants with better educational levels showed better control ( $\mathrm{p}$ value $=0.005$ ). However, no significance was found between HbA1c and age, BMI, following a specific diet and the time since diabetes diagnosis. During history taking interview with the patients, $72(63.7 \%)$ cases declared never meeting a dietitian. Eighty eight (77.9\%) cases said they practiced exercise out of whom $82(72.6 \%)$ specified walking and $2(1.8 \%)$ mentioned swimming. The mean knowledge score was found out to be 8.7 out of 14 .

Table 1:- Table 1 shows frequency and percentage of age, weight, gender, marital status, job and education of the diabetes mellitus type-2 patients.

\begin{tabular}{|l|l|l|}
\hline Variables & Frequency & Percentage \\
\hline Age Group & & \\
$20-39$ & 8 & $7.14 \%$ \\
$40-59$ & 70 & $61.50 \%$ \\
$60-79$ & 33 & $29.46 \%$ \\
$80-99$ & 1 & $.89 \%$ \\
\hline Weight Group & 9 & \\
$18.5-24.9$ & 30 & $8.04 \%$ \\
25.29 .9 & 38 & $26.79 \%$ \\
$30-34.9$ & 21 & $33.93 \%$ \\
$35-39.9$ & 14 & $18.75 \%$ \\
$>=40$ & & $12.50 \%$ \\
\hline Gender & 64 & \\
Male & 48 & $57.14 \%$ \\
Female & & $42.86 \%$ \\
\hline Residence & 104 & \\
Urban & 8 & $92.86 \%$ \\
Rural & & $7.14 \%$ \\
\hline Marital Status & 9 & \\
Not married & 103 & $8.04 \%$ \\
Married & & $91.69 \%$ \\
\hline Job & 76 & $67.86 \%$ \\
Not working & 23 & $20.54 \%$ \\
Office & 13 & $11.61 \%$ \\
Field & & \\
\hline Education & 32 & $28.57 \%$ \\
Not educated & 27 & $24.11 \%$ \\
Elementary/Secondary & 25 & $22.32 \%$ \\
High school/Diploma & 28 & $25.00 \%$ \\
University or above & & \\
\hline
\end{tabular}


Table 2:- Table shows combined results of all aspects of the study.

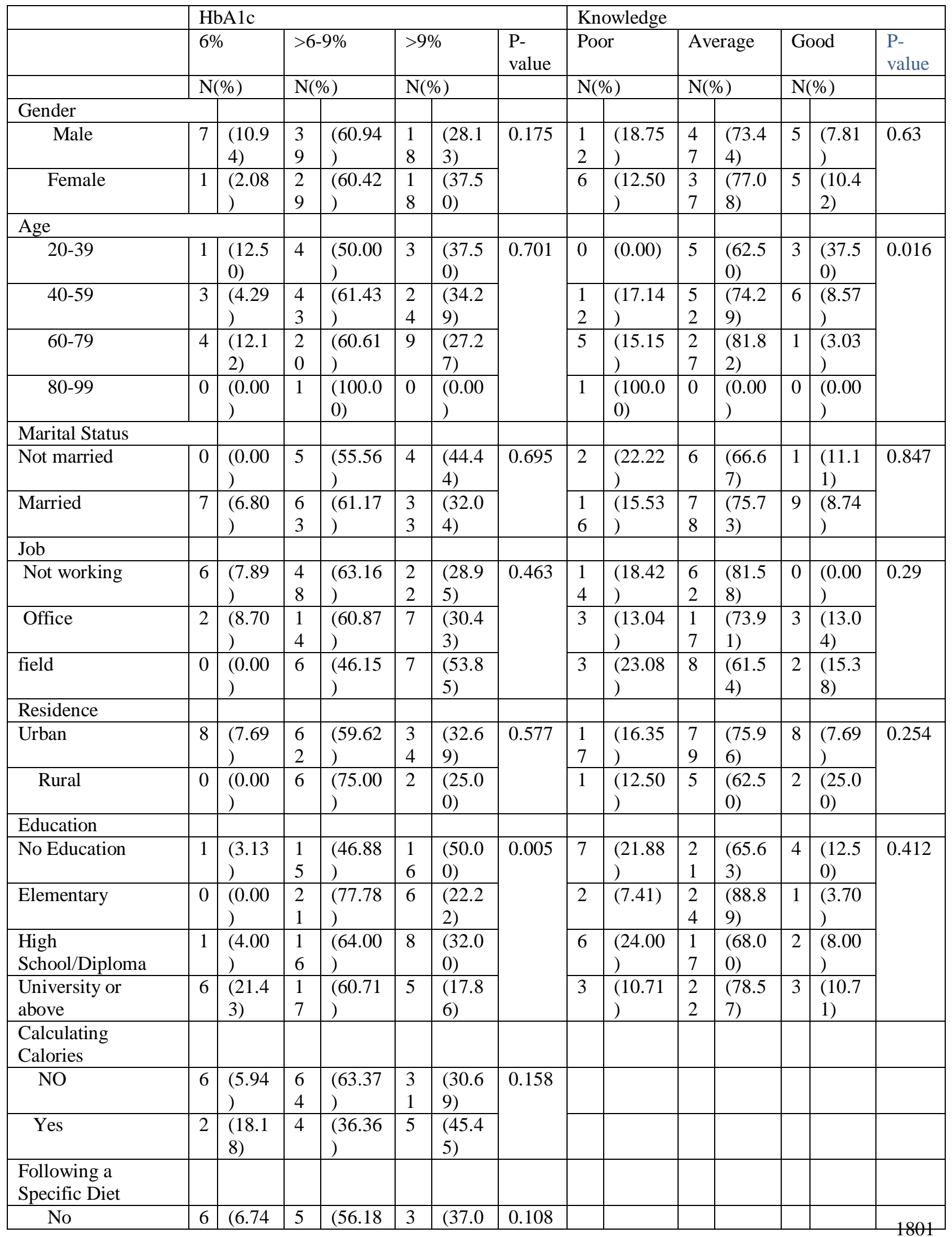




\begin{tabular}{|l|l|l|l|l|l|l|l|l|l|l|l|l|l|l|}
\hline & & ) & 0 & ) & 3 & $8)$ & & & & & & & & \\
\hline Yes & 2 & $(8.70$ & 1 & $(78.26$ & 3 & $(13.0$ \\
& & & & & & & & & & \\
\hline
\end{tabular}

Table 3:- Mean and median of attitude items (questions with reverse scoring)

\begin{tabular}{|c|c|c|c|}
\hline & \multicolumn{2}{|c|}{ Mean(SD) } & Median \\
\hline Diabetes patients should monitor carbohydrates consumption & 4.46 & $(0.80)$ & 5.00 \\
\hline Diabetes patients should consult dietitians & 4.63 & $(0.63)$ & 5.00 \\
\hline Diabetes patients should follow a certain diet designed specifically for them & 4.48 & $(0.73)$ & 5.00 \\
\hline Overweight diabetes patients should lose weight & 4.77 & $(0.44)$ & 5.00 \\
\hline Hydration leads to lower glucose levels and better diabetes control & 4.40 & $(0.79)$ & 5.00 \\
\hline Physical activities improve diabetes control & 4.77 & $(0.46)$ & 5.00 \\
\hline Diabetes patients should exercise & 4.86 & $(0.35)$ & 5.00 \\
\hline Smoking increases the risk of diabetes complications & 4.52 & $(0.76)$ & 5.00 \\
\hline
\end{tabular}

\section{Discussion:-}

Our study summarized the attitude, knowledge and life style of diabetic type-2 patients residing in Saudi Arabia for diabetic control and HbA1c results. The basis for our study was the implementation of modification of lifestyle, knowledge increment and to study their attitude towards diabetes and evaluating the results for better control of the disease. However, total knowledge score did not seem to affect HbA1c level, which probably indicates a huge lack of implementation of the knowledge in everyday lifestyle practices.

Poor control of HbA1c among our subjects is also supported by another study that was conducted in Makkah, Saudi Arabia, in which the mean HbA1c of the subjects was 7.3 that poor control by those cases (21).

In terms of physical exercise, most subjects reported only walking which is similar to the findings in one of the studies, which was conducted in Jamshoro, Pakistan (17). Females turned out to be significantly doing more exercise than malesalthough the intensity of exercise does not fulfill the ADA (American Diabetes Association) criteria for exercise for both.

As for visiting dietitians for dietary advice, our results are alarming since $63.7 \%$ of our subjects never met a dietitian. This is even worse than what was found in another study in the United Arab Emirates (14) in which the number of such people was $46 \%$ which still shows that a huge proportion of diabetics are not aware of such provisions.

With the results of our study we can figure out the knowledge and lifestyle of diabetic patients and their attitude towards it. Our study can be regarded as a good study because of the fact that the study was conducted in a tertiary care hospital and anthropometric and biochemical parameters information were taken from the hospital records and there was no follow up.

\section{Conclusion:-}

In conclusion, our study showed that type 2 diabetes mellitus patients in Saudi Arabia have an average amount of knowledge about required lifestyle changes. Attitude to lifestyle changes was extremely good in almost all cases, however exercise among most study individuals turned out to be not so good. This indicates a lack of will-power for the correct implementation of their knowledge and attitude. As for HbAlc, levels in the vast majority of cases showed poor control on the disease.

We would recommend the development of easy to use software for the calculation of calories. Such software would make it easier for patients to follow their daily calorie intake and comply with their specified diet regimens. It would be also very important to emphasize the importance and the role of dietitians in managing diabetes. We would also recommend that all restaurants and food suppliers specify the amount of calories in each meal to help in calculating calories which makes it easy to maintain a healthy diet. An additional important point would be an implementation a healthy diet which suits both normal and diabetic individuals. Our last suggestions to construct safe sidewalks for diabetics to be able to exercise more frequently. 


\section{Study limitations:-}

There are some limitations in our study.One of the limitations is the short period in which the study was conducted, along with our heavy study load. Another limitation was our inability to collect data from female primary care clinic, which resulted in difficulty in obtaining proper data from the female side. Limited sample size made us unable to generalize our study to whole population of Saudi Arabia.

\section{Acknowledgment:-}

We would like to thank for the help we got from the family and community medicine department in King Khaled University Hospital regarding our research methodology and statistical analysis. We would like to appreciate the help of the female medical students (Shaza Nasser Almweisheer, Alanood Ahmed Asiri and Tarfah Fahad Alobaidan) in collecting the data from female subjects for our study. We are grateful to Tusneem Elhassan, from KFSH\&RC who helped us in statistics. Finally, we appreciate the help of all health care workers in the primary care clinic for their cooperation throughout our study

\section{References:-}

1. Wu Y1, Ding Y1, Tanaka Y2, Zhang W3. Risk factors contributing to type 2 diabetes and recent advances in the treatment and prevention. Int J Med Sci. 2014 Sep 6; 11(11):1185-200.

2. IDF Diabetes Atlas 7th edition. 2015. http://www.diabetesatlas.org/

3. International Diabetes Federation, IDF Diabetes Atlas, International, Diabetes Federation, Brussels, Belgium, 6th edition, 2013, http://www.idf.org/diabetesatlas.

4. Al-Rubeaan K, Al-Manaa HA, Khoja TA, Ahmad NA, Al-Sharqawi AH, Siddiqui K, Alnaqeb D, Aburisheh KH, Youssef AM, Al-Batel A, Alotaibi MS, Al-Gamdi AA. Epidemiology of abnormal glucose metabolism in a country facing its epidemic: SAUDI-DM study. J Diabetes. 2015 Sep;7(5):622-32. doi: 10.1111/1753-0407.12224. Epub 2014 Dec 1.

5. Arab M. The economics of diabetes care in the Middle East. In Alberti K, Zimmet P, Defronzo R, editors. International Textbook of Diabetes Mellitus. Second Edition. Chichester: John Wiley and Sons Ltd; 1997.

6. World Bank. World Bank Data, WHO parameters, 1999-2000. World Bank; 2000.

7. Schramm TK, Gislason GH, Køber L et al. Diabetes patientsrequiring glucose-lowering therapy and non-diabetics with aprior myocardial infarction carry the same cardiovascular riska population study of 3.3 million people. Circulation. 2008;117(15): 1945-1954.

8. Rhee MK, Slocum W, Ziemer DC, Culler SD, Cook CB,El-Kebbi IM, Phillips L S. Patient adherence improvesglycemic control. The Diabetes Educator. 2005; 31(2):240-250.

9. http://www.who.int/mediacentre/factsheets/fs310/en/

10. Upadhyay DK, Palaian S, Shankar PR, Mishra P. Knowledge, attitude and practice about diabetes among diabetes patients in western Nepal. Rawal Med J. 2008;33 (1):8-11.

11. Gunay T, Ulusel B, Velipasaoglu S, Unal B, Ucku R, Ozgener N. Factors affecting adult knowledge of diabetes in Narlidere Health District, Turkey. ActaDiabetologica. 2006;43:142- 147.

12. Khattab MS1, Aboifotouh MA, Khan MY, Humaidi MA, al-Kaldi YM. Compliance and control of diabetes in a family practice setting, Saudi Arabia. East Mediterr Health J. 1999 Jul;5(4):755-65.

13. Abahussain NA1, El-Zubier AG. Diabetes knowledge among self-reported diabetic female teachers: Al-khobar, saudiarabia.Abahussain NA1, El-Zubier AG.J Family Community Med. 2005 Jan;12(1):43-8.

14. Al-Kaabi J, Al-Maskari F, Saadi H, Afandi B, Parkar H, Nagelkerke N. Assessment of dietary practice among diabetic patients in the United arabemirates. Rev. Diabet. Stud. RDS. Society for Biomedical Diabetes Research; 2008;5(2):110.

15. Elkharam WM, Khatri R, Wallymahmed AH, Gee IL, Elhisadi T. Knowledge of and Adherence to Health Advice among Adults with Diabetes in Libya. Ibnosina J. Med. Biomed. Sci. 2013;5(3):140-7.

16. Al-Adsani AMS, Moussa MAA, Al-Jasem LI, Abdella NA, Al-Hamad NM. The level and determinants of diabetes knowledge in Kuwaiti adults with type 2 diabetes. Diabetes Metab. Elsevier; 2009;35(2):121-8.

17. ShaikhZali, Ali G, Shaikh MZ. Diabetec patients; awreness about life style modifications. Professional Med J AprJun 2011;18(2): 265-268.

18. VanVoorhis CRW, Morgan BL. Understanding power and rules of thumb for determining sample sizes. Tutor. Quant. Methods Psychol. 2007;3(2):43-50.

19. Fitzgerald JT, Funnell MM, Hess GE, Barr PA, Anderson RM, Hiss RG, et al. The reliability and validity of a brief diabetes knowledge test. Diabetes Care. Am Diabetes Assoc; 1998;21(5):706-10.

20. Khan H, Sobki S, Khan S. Association between glycaemic control and serum lipids profile in type 2 diabetic patients: HbA1c predicts dyslipidaemia. Clinical and experimental medicine. 2007;7(1):24--29.

21. Abdullah Almarzouki, EmaadTashkandi, Mian Farooq, Glycated Hemoglobin and 'A1c' Complications, Am. Biomed. Sci. 2012, 4(2), 153-156. 